\title{
Ureteroscopic Laser Lithotripsy: A Review of Dusting vs Fragmentation with Extraction
}

\author{
Brian R. Matlaga, MD, MPH, Ben Chew, MD,2 Brian Eisner, MD, Mitchell Humphreys, MD, \\ Bodo Knudsen, MD, Amy Krambeck, MD, Dirk Lange, PhD, Michael Lipkin, MD, Nicole L. Miller, MD?, \\ Manoj Monga, MD, Vernon Pais, MD, Roger L. Sur, MD, ${ }^{10}$ and Ojas Shah, MD ${ }^{11}$ (EDGE Research Consortium)
}

\begin{abstract}
Introduction: Ureteroscopic laser lithotripsy is becoming the most commonly utilized treatment for patients with urinary calculi. The Holmium:YAG (yttrium aluminum garnet) laser is integral to the operation and is the preferred flexible intracorporeal lithotrite. In recent years, there has been increasing interest in examining the effect of varying the laser settings on the effectiveness of stone treatment. Herein, we review the two primary laser treatment approaches: dusting and fragmentation with extraction.

Methods: We reviewed PubMed and MEDLINE databases from January 1976 through January 2017. All authors participated in the development of consensus definitions of dusting and fragmentation with extraction. The review protocol adhered to preferred reporting items for systematic reviews and meta-analyses (PRISMA) methodology.

Results: When the Holmium:YAG laser is used to treat stones, there are two parameters that can be adjusted: power $(\mathrm{J})$ and frequency $(\mathrm{Hz})$. In one treatment paradigm, which became termed "fragmentation with extraction," laser settings that relied on high energy and low frequency were used. Another paradigm, which became termed "dusting," utilized low energy and high frequency settings, which had the effect of breaking off exceedingly small fragments from the stone.

Conclusions: Both dusting and fragmentation with extraction approaches to ureteroscopic stone treatment are effective. In fact, there is little evidence that one approach is better than the other. However, each does have relative advantages and disadvantages, which should be considered. Although dusting tends to be associated with shorter procedure times and a lower risk of ureteral damage, this approach may place the patient at increased risk for future stone events should all of the resultant debris not be expelled from the collecting system. The active removal associated with fragmentation with extraction, in contrast, may provide for a more complete initial stone clearance.
\end{abstract}

Keywords: ureteroscopy, lithotripsy, laser

\section{Introduction}

$\mathbf{H}$ OLMIUM: YAG (yttrium aluminum garnet) LASER lithotripsy induces stone fragmentation through the direct absorption of energy by the calculus. Laser energy is transmitted to the stone surface by means of direct energy transfer and an accompanying vapor bubble that is created at the fiber tip during activation of the laser. The collapse of the vapor bubble may also produce a weak acoustic effect. ${ }^{1,2}$ The pulse energy settings for the Ho:YAG laser differ from 0.2 to $2.0 \mathrm{~J}$ depending on the manufacturer and model of laser console used. Lower pulse energy settings result in smaller stone

\footnotetext{
${ }^{1}$ James Buchanan Brady Urological Institute, Johns Hopkins Medical Institutions, Baltimore, Maryland.

${ }^{2}$ Department of Urologic Sciences, University of British Columbia, Vancouver, British Columbia.

${ }^{3}$ Department of Urology, Massachusetts General Hospital, Boston, Massachusetts.

${ }^{4}$ Department of Urology, Mayo Clinic, Indianapolis, Indiana.

${ }^{5}$ Division of Urology, Department of Surgery, Ohio State University Medical Center, Columbus, Ohio.

${ }^{6}$ Department of Surgery and Urology, Duke University Medical Center, Durham, North Carolina.

${ }^{7}$ Department of Urologic Surgery, Vanderbilt University School of Medicine, Nashville, Tennessee.

${ }_{9}^{8}$ Cleveland Clinic Foundation, Cleveland, Ohio.

${ }^{9}$ Department of Urology, Dartmouth Hitchcock Medical Center, Lebanon, New Hampshire.

${ }^{10}$ Department of Surgery/Urology, UC San Diego, San Diego, California.

${ }^{11}$ Department of Urology, NYU, New York, New York.
} 
fragments and less retropulsion. ${ }^{3}$ As the pulse energy is increased, fragment size increases, as does the degree of retropulsion.

The pulse frequency setting (pulses/second/Hz) can range from $\sim 4$ to $80 \mathrm{~Hz}$ depending on the model of Ho:YAG laser. The frequency governs how many pulses of laser energy are delivered to the stone per second. Higher pulse frequency will result in faster lithotripsy time provided that the tip of the laser fiber remains in good contact with the target stone.

\section{Methods}

We reviewed PubMed and MEDLINE databases from January 1976 through January 2017. All authors participated in the development of consensus definitions of dusting and fragmentation with extraction. The review protocol adhered to preferred reporting items for systematic reviews and metaanalyses (PRISMA) methodology. PubMed, Embase, and Google Scholar were also queried for studies relating to "ureteroscopy" and "lithotripsy." There was no limit with regards to the year of publication, and all identified articles were included in the final aggregation. Due to the heterogeneity of the original research, and limited volume of publications, a formal meta-analysis was precluded. There were three randomized controlled trials identified, but methodology was heterogeneous, as were the populations studied.

\section{Evolution of laser settings for lithotripsy}

Early in the introduction of Holmium laser lithotripsy, it was noted that altering the treatment parameters affected the efficiency of fragmentation and risk of retropulsion. ${ }^{4}$ For example, low pulse energy $(0.2 \mathrm{~J})$ led to smaller fragments and less retropulsion at the cost of fragmentation efficiency. ${ }^{3}$ Some Holmium laser devices can also provide the ability to modulate the pulse duration. A longer laser pulse duration (700 or $1500 \mu$ seconds) compared to a traditional pulse duration ( 300 or $350 \mu$ seconds) has been demonstrated in in vitro studies to provide effective stone comminution while reducing laser fiber tip degradation and stone retropulsion. ${ }^{5,6}$ To maximize efficiency of Holmium laser lithotripsy, shorter pulse durations with higher pulse energy have been recommended. $^{7}$ A comprehensive analysis confirmed that low frequency $(5 \mathrm{~Hz})$ and high pulse energy $(1.2 \mathrm{~J})$ lead to more efficient stone ablation as manifested by deeper and wider stone fissures. ${ }^{8}$

\section{Dusting}

Surgical technique for dusting. The goal of dusting is to fragment the stone into tiny pieces that resemble "dust" and thus can pass spontaneously. This is accomplished by utilizing the Holmium laser at a high frequency (HF) with low energy (Table 1). The technique of dusting involves working tangentially from the edge of the stone with the laser fiber taking care not to break off large fragments from the main stone. Laser settings in this situation are crucial to help produce smaller pieces of dust. As the procedure progresses, the surgeon should continue to treat the edges of the stone circumferentially or from one leading edge.

The other principle involved in dusting is to keep the laser fiber slightly off of the stone to "defocus" it and minimize
Table 1. Typical Laser Settings for Dusting VS Fragmentation With Extraction

\begin{tabular}{lc}
\hline $\begin{array}{l}\text { Dusting; } \\
\text { HF/low energy }\end{array}$ & $\begin{array}{c}\text { Fragmentation with extraction; } \\
\text { low frequency/high energy }\end{array}$ \\
\hline Frequency & \\
$\quad 12-15 \mathrm{~Hz}(<30 \mathrm{~W}$ laser $)$ & $6-10 \mathrm{~Hz}$ \\
$\quad 50 \mathrm{~Hz}(100 / 120 \mathrm{~W}$ laser $)$ & \\
Energy & \\
$\quad 0.2-0.4 \mathrm{~J}$ & $1.0-1.2 \mathrm{~J}$ \\
\hline
\end{tabular}

$\mathrm{HF}=$ high frequency.

the mechanical acoustic effect of the laser energy. Defocusing the laser will produce smaller fragments (i.e., dust). Accounting for respiratory renal movement is also important. Fine movements of the hand holding the tip of the ureteroscope as it enters into the urethra or a fine movement of the thumb-activated deflecting cables will allow the surgeon to "paint" the stone from the edge toward the middle of the stone. At the beginning of the case, the laser energy is typically less than the mass of the stone and the stone does not tend to move much and stays in place. Once the stone is ablated down to a smaller mass, the laser energy becomes greater than the stone fragment and the stone will start to bounce within the calix making it more difficult to shave the stone from the edges. When this happens, one can either extract the remaining pieces or place the laser fiber safely in the middle of the calix and keep discharging the laser as fragments come into contact with the fiber-a technique known as "popcorning."

Several visual cues may suggest that the fragments are small enough to pass. The most commonly used technique is to measure the size of the fragments against the diameter of the laser fiber that is being used in the ureteroscope. Second, if a few pieces are extracted, these can be judged and measured once they are removed from the body.

\section{Rationale for dusting}

Proponents of the dusting technique may concede that immediate postoperative stone-free rates may not be improved with this technique-certainly active stone retrieval with basket extraction could result in patients having fewer fragments postoperatively than dusting techniques, which pulverize stones into such tiny fragments that basket extraction is not possible. However, a recent prospective multicenter study of dusting $v s$ fragmentation with stone retrieval for renal stones $\leq 15 \mathrm{~mm}$ in diameter demonstrated that in the short term, there was no difference in readmission rates, reintervention rates, or patients becoming symptomatic from residual fragments. ${ }^{9}$ An additional recent study, examining ureteroscopy with lithotripsy for stones $10-40 \mathrm{~mm}$, noted that active fragment retrieval using a Nitinol basket was not associated with improvements in stone-free rates. ${ }^{10}$

Dusting may also confer advantages in patient outcomes by reducing the potential for postoperative complications. The lower power, higher frequency settings used for dusting are associated with decreased stone retropulsion during stone fragmentation. ${ }^{11}$ Decreased stone movement and retropulsion may allow for more efficient ureteral stone treatment by minimizing the need to "chase" ureteral stones, which 
have migrated cephalad. Other studies have also demonstrated that stone retropulsion may increase the need for secondary procedures to treat clinically significant stone fragments which have migrated from the ureter to the proximal ureter or kidney. ${ }^{12}$

Dusting may be associated with shorter operative times due to omission of active fragment retrieval. Two recent studies have demonstrated $40 \%$ and $20 \%$ reductions in operative times associated with the dusting technique., ${ }^{9,13}$ Another potential advantage for dusting is decrease in use of ureteral access sheaths, which may lead to decreases in ureteral trauma. The initial cohort for the Post-Ureteroscopic Lesion Scale study reported that ureteral access sheath was associated with some ureteral trauma in $57 \%$ of patients (superficial or submucosal lesion). ${ }^{14}$ It should be noted, although, that the relationship between such ureteral trauma and subsequent ureteral stricture formation is not yet well defined. It has also been reported that basket-associated complications are reduced with dusting. ${ }^{13}$ In addition, as there is less of a need for multiple implements to be passed through the ureteroscope's working channel, there may be greater scope longevity with this approach.

A final argument for dusting involves the cost of the procedure. Both disposable instruments used for flexible ureteroscopy and operating room costs should be taken into account. If one considers laser lithotripsy with and without active fragment retrieval, both procedures will require a guidewire, as well as a laser fiber. However, a dusting procedure can often be accomplished with those devices alone. Extraction, although, may require use of a ureteral access sheath and a grasper or basket, and in some cases, the use of a safety wire to maintain access to the ureter while fragments are being extracted. In addition, as described above, dusting is associated with $20 \%-40 \%$ reduction in operative times in two reports. The cost of operating room time is estimated as \$29-\$80/minute, and therefore, dusting may significantly reduce operating room costs. However, studies have not taken into account the potential cost of a secondary procedure for symptomatic residual fragments, which should also be considered. $^{15}$

\section{Clinical outcomes of dusting}

A number of recent studies have looked at the outcomes of in vivo renal stone dusting. Several of these have been retrospective, which should be noted as such studies have a risk for selection bias. A retrospective study evaluating dusting of intrarenal stones using settings of $0.2-0.5 \mathrm{~J}$ and $30-80 \mathrm{~Hz}$ reported a stone-free rate of $67.2 \% .^{16}$ The mean stone size of the cohort was $11.5 \mathrm{~mm}$ (range $5-50 \mathrm{~mm}$ ). Of the 71 patients, 4 presented to the emergency department postop, but did not require admission. The complication rate was $14 \%$, although all were either Clavien Grade 1 or 2 . Another review by the same group compared dusting using 60-100 W Holmium lasers with dusting using the $120 \mathrm{~W}$ Holmium laser. ${ }^{17}$ Settings of $0.2-0.5 \mathrm{~J}$ and $30-50 \mathrm{~Hz}$ were used with the lower powered lasers, and in the group treated with the $120 \mathrm{~W}$, the authors used settings of $0.2-0.5 \mathrm{~J}$ and $30-80 \mathrm{~Hz}$. The stonefree rate for the group treated with the $120 \mathrm{~W}$ laser was $66 \%$ vs 39\% $(p<0.05)$ for those treated with the 60-100 W laser. The authors concluded that ultra-HF $(80 \mathrm{~Hz})$ improves stonefree rates with dusting.
Another group compared low power (LP)/HF laser settings $(0.2-0.4 \mathrm{~J}$ and $50 \mathrm{~Hz})$ with conventional laser settings $(0.5-$ $1.0 \mathrm{~J}$ and $5-10 \mathrm{~Hz}$ ) for the treatment of intrarenal stones. ${ }^{18}$ The mean stone burden was $16.5 \mathrm{~mm}$ for the LP/HF group and $10 \mathrm{~mm}$ for the conventional group. The operative times were similar for the two groups. Interestingly, complete stone fragmentation to fine dust obviating the need for basket extraction was only accomplished in $13.4 \%$ of the LP/HF cases.

\section{Fragmentation with extraction}

Surgical technique of fragmentation with extraction. Laser setting requirements for fragmentation with extraction are actually the "typical" laser settings that have been used since the introduction of Ho:YAG lithotripsy (Table 1). Given the constraints of early Holmium laser technology, urologists routinely would use combination energy settings such as $0.6-1.0 \mathrm{~J}$ along with rates of $6-10 \mathrm{~Hz} .{ }^{1}$ Furthermore, in vitro studies also confirm that stone fragmentation size is proportional to pulse energy. ${ }^{2}$

In general, fragmentation with extraction relies on higher pulse energy (such as $0.6-1.2 \mathrm{~J}$ ) and lower frequency $(6-10 \mathrm{~Hz}$ ). In vitro as well as clinical practice has demonstrated that these settings create stone fragments that are larger and ideal for removing with extraction devices. A common technique is to begin with a relatively low energy and rate setting (e.g., $0.6 \mathrm{~J}, 6 \mathrm{~Hz}$ ) and only increase if necessary. This technique not only maximizes laser tip preservation but also minimizes retropulsion or stone movement during lithotripsy.

In the setting of difficult ureteral access to proximal ureteral or renal stones, placement of a ureteral access sheath may expedite access, fragmentation, and subsequent clearance of the targeted stone. ${ }^{19-21}$ However, based upon surgeon experience an access sheath is not mandatory in an appropriately capacious ureter. The access sheath may in fact limit the size of stone fragments that can be retrieved, and thus, judicious omission may be considered.

The mid-portion of the stone is typically targeted first, with the goal of breaking the stone into halves. Beginning in the mid-portion of the stone additionally maximizes the margin of safety between the laser fiber and the ureteral or renal wall. The generated fragments can then be sequentially further fragmented in the same manner until they appear to be of a retrievable size. A small caliber Nitinol grasper or basket is used to affect stone clearance. Active extraction continues until all visible fragments have been cleared.

\section{Rationale for fragmentation with extraction}

The process of fragmenting and retrieving kidney stones may hold several advantages. Stone composition may direct metabolic intervention to help prevent future stone episodes; absence of this information may limit the specificity of patient counseling. If residual stone material is left behind at the time of initial stone treatment, patients may also be at increased risk for future stone events. Chew and colleagues looked at the natural history of asymptomatic stone fragments left behind after ureteroscopy (URS) and found that only $56 \%$ of patients required no intervention and remained asymptomatic. Intervention was required in $29 \%$ of patients, with a greater risk associated with larger fragments leading them to conclude that complete stone-free status is the most effective strategy to reduce stone events following ureteroscopy. ${ }^{22}$ 


\section{Clinical outcomes of fragmentation with extraction}

When reporting stone-free outcomes for flexible ureteroscopic stone extraction, one must consider stone-free rates, imaging used to determine stone-free rates, safety, and cost. The definition of stone free is not standardized and differs greatly in the literature. Some studies report "stone free" as absolutely no calcifications noted on the postoperative imaging. Other investigators include patients with fragments $<2 \mathrm{~mm}$ in the "stone free" definition, while others yet include fragments $\leq 5 \mathrm{~mm}$ as "stone free," citing that such fragments are clinically insignificant. Timing of stone-free determination is also not standardized. Since fragments may pass from the kidney for a period of time after ureteroscopic procedures, it is debatable if "stone-free" outcomes should be measured within days, weeks, or months of the procedure. The type of postoperative imaging is also important as sensitivity to detect small fragments is greatest with $\mathrm{CT}$, intermediate with plain abdominal X-rays (kidney, ureter, and bladder radiograph [KUB]), and least sensitive with ultrasound (US). Safety can be reported as immediate intraoperative complications or may be expanded to include complications as much as 90 days postprocedure. Finally cost not only includes cost of the surgical devices but also operating room time and days lost from income for the patient. Such varied definitions make interpretation and comparison of flexible ureteroscopy studies difficult.

When discussing cost, active basket extraction typically increases immediate cost, as it requires the use of a disposable basket or grasper and generally an access sheath. Furthermore a recent study from the EDGE consortium noted a decrease in operating room (OR) time with the dusting procedure even for larger stones, which would lower cost associated with OR time usage. ${ }^{9}$ Conversely the same study, which was a prospective evaluation, demonstrated a decreased likelihood of significant residual fragments when basket extraction was performed, which most likely translates into decreased patient cost over the long term. Fragments $>4 \mathrm{~mm}$ were associated with an increased risk of intervention, while fragments $>2 \mathrm{~mm}$ were associated with stone growth overtime. Further studies will be needed to determine if the immediate cost savings noted up front with dusting outweighs the long-term cost risks of repeat intervention and time lost for the patient.

\section{Comparison studies}

Despite the fact that dusting and fragmentation with extraction techniques for stone treatment have been routinely utilized for years, there is a paucity of comparative studies. Gamal and Mamdouh conducted a study of 46 patients with unilateral renal stones less than $2 \mathrm{~cm}$ undergoing flexible ureteroscopy. ${ }^{13}$ Patients were randomized into two groups: Group $1(n=23)$ in which the stone was dusted using LP (.2$0.4 \mathrm{~J})$ and $\mathrm{HF}(20-30 \mathrm{~Hz})$, and Group $2(n=23)$ in which the stone was fragmented using high power (1-2 J) and low frequency $(4-5 \mathrm{~Hz})$ with basket extraction of fragments. Stone-free rates were similar between the two groups (86\% vs $89 \%)$. Dusting was associated with significantly less operative time (57 minutes vs 70 minutes, $p=0.001)$. Ureteral access sheaths were used only in the patients in Group 2.

The EDGE research consortium performed a more recent study, which was of a multicenter, prospective cohort design conducted at eight centers specializing in high-volume, complex endourologic stone treatment. ${ }^{9}$ Surgeons who primarily performed fragmentation with extraction procedures included their patients in the "basketing" arm (five centers), and surgeons who routinely dust stones recruited patients for that arm (three centers). A standardized protocol was used for preoperative evaluation, follow-up imaging, and postoperative care, including ureteral stent placement in all patients and an alpha-blocker for 30 days. The definition of stone free was no fragments of any size on KUB and/or US imaging within 3 months. One hundred fifty-two patients with radiopaque renal stones from 5 to $20 \mathrm{~mm}$ were included in the study with 70 in the dusting arm and 82 in the basketing arm. The stones were slightly larger in the dusting group $122.6 \pm 88.6 \mathrm{~mm}^{2}$ vs the basketing group $82.3 \pm 82.3 \mathrm{~mm}^{2}$. Laser energy used was higher in the dusting arm $(46.4 \mathrm{~kJ} v s 22.8 \mathrm{~kJ})$. Ureteral access sheaths were used in $100 \%$ of patients in the basketing group and only $18.2 \%$ of those in the dusting group. Operative time was longer in the basketing arm. The stone-free rate was significantly higher for patients in the basketing arm, $86.3 \%$, compared to $59.2 \%$ in the dusting arm. There was no difference in readmission to emergency room (ER) or hospital between the groups (14.5\% dusting vs $14 \%$ basketing) or in reintervention rates (two patients in dusting, three in basketing). There were no differences in stone composition or complication rate between the groups. In summary, this study found a higher stone-free rate for basketing compared to dusting; however, this comes at the expense of longer OR time and perhaps greater procedural cost as a ureteral access sheath was routinely utilized.

Lee and colleagues published a retrospective study comparing patients undergoing ureteroscopy for renal stones who had stone fragments actively basketed (Group A, $n=172$ ) to those whose stones were fragmented with laser lithotripsy alone (Group B, $n=76$ ). ${ }^{23}$ It is important to note that the group of patients who underwent lithotripsy alone may not have been treated with a "dusting" approach as the laser settings were not reported. Mean stone size was $11.1 \mathrm{~mm}$ for both groups. Operative time was also similar (82.5 minutes for basketing and 82.1 minutes for dusting). Success rate was determined by CT imaging within 1 month postoperatively and was defined as stone free or with clinically insignificant fragments $(<3 \mathrm{~mm})$. There was no difference between the groups in success rates $(89 \%$ vs $86.8 \%)$ or complication rate (9.9\% vs $11.8 \%)$.

Two published studies have evaluated dusting $v s$ basketing for treatment of ureteral stones. The first by Schatloff and colleagues randomized 60 patients with ureteral stones to active basket retrieval of fragments vs spontaneous passage of fragments following laser lithotripsy. ${ }^{24}$ Imaging to determine stone-free rates consisted of ultrasonography and KUB within 1 month postprocedure for patients with radiopaque stones and CT for those with radiolucent stones. The stonefree rate for basketing was higher than for dusting, $100 \% \mathrm{vs}$ $87 \%$, but this was not statistically significant $(p=0.10)$. The patients randomized to the dusting arm had a higher rate of unplanned medical or emergency room visits within the 30day postoperative period (30\% vs 3\%, OR 12.4).

The second study conducted by Fahmy and colleagues compared dusting $v s$ basketing during semirigid ureteroscopic laser lithotripsy in children. One hundred children (age 1-15 years) with symptomatic ureteral stones were prospectively randomized to one of two groups: dusting $(n=50)$ or basketing $(n=50) .{ }^{25}$ Laser settings utilized for dusting were $0.5 \mathrm{~J}$ and $12-$ 
Table 2. Advantages and Disadvantages of Laser Lithotripsy Approaches

\begin{tabular}{|c|c|}
\hline Advantages & Disadvantages \\
\hline \multicolumn{2}{|l|}{ Dusting } \\
\hline Shorter procedure time & No stone material for analysis \\
\hline Reduced risk of ureteral trauma & Retained stone material (assumes spontaneous fragment passage) \\
\hline $\begin{array}{l}\text { Fewer consumable devices required } \\
\text { (i.e., no access sheath, basket, or grasper) }\end{array}$ & May not be effective for all stone compositions \\
\hline \multicolumn{2}{|l|}{ Fragmentation with extraction } \\
\hline Nothing for patient to pass & Longer procedure time \\
\hline $\begin{array}{l}\text { Stone analysis may inform metabolic } \\
\text { management }\end{array}$ & $\begin{array}{l}\text { More consumable device required (i.e., access sheath, } \\
\text { basket/grasper) }\end{array}$ \\
\hline Immediate stone-free rates superior to dusting & $\begin{array}{l}\text { Technically more intensive for urologist (repeated ureteroscopic } \\
\text { passages, requires skilled assistant) }\end{array}$ \\
\hline
\end{tabular}

$15 \mathrm{~Hz}$, while those used for fragmentation with subsequent basket retrieval were $1-1.5 \mathrm{~J}$ and $8-10 \mathrm{~Hz}$. Stone free was assessed by US and KUB 1 week following the procedure and defined as absence of any stone fragments. The operative time was less in the basketing group (28 minutes vs 36 minutes, $p=0.006)$. However, stone-free rates were similar (98\% dusting, $96 \%$ basketing). There was also no difference in complications all of which were classified as minor: transient hematuria and urinary tract infection (UTI).

The comparison of dusting and fragmentation with extraction techniques for ureteral stones is undoubtedly impacted by stone location and size. One might reason that dusting a larger proximal or mid-ureteral stone in a hydronephrotic ureter may be less efficacious as stone retropulsion and fragment migration are common. Although both studies found a benefit of basket stone extraction over dusting, additional studies with stratification by stone size and location and laser settings are necessary to support these conclusions.

\section{Clinical application of dusting and fragmentation with extraction}

The question of which surgical technique is optimal for treating renal stones remains controversial and should be determined on a case-by-case basis.

Fragmentation with extraction is appealing for both the surgeon and patient for many reasons (Table 2). Theoretically, by the end of the surgery, patients should be close to stone free and not need to pass fragments. Surgeons will also have a stone sample available for analysis, which will help in guiding future metabolic therapy and also providing dietary recommendations. However, active extraction typically increases immediate cost, as it usually has longer operative times, requires use of an extraction device, and utilizes a ureteral access sheath.

Dusting by fragmenting stones into tiny particles $<1-$ $2 \mathrm{~mm}$ theoretically decreases operative time and the risk of ureteral trauma by negating the need for use of a ureteral access sheath (UAS). In addition, dusting reduces the immediate procedure cost by eliminating the need for a stone retrieval device, possibly a ureteral stent in uncomplicated cases, as well as additional staff, since the surgeon can typically perform the procedure with little to no assistance. However, potential risks exist with the dusting technique, such as recurrent stone formation from fragments/dust failing to pass, as well as an increased risk of secondary interventions and ER visits by patients passing stones. Until long-term data are available, it is not clear if it is better to have residual dust or a residual fragment in select cases.

An optimal approach may be some combination of the techniques based on numerous stone factors, including size, location, and density, as well as each patient's anatomic features. The complete eradication of stone fragments is one of the primary outcomes of ureteroscopy, but this aim must not come at the expense of increased risk of complications for the patient. Thus, urologists must be cognizant of treatment options to personalize care, as they can be used differently in the myriad of clinical scenarios that exist. At present, based on our findings, the dusting $v s$ fragmentation with extraction discussions appear to be limited to flexible ureteroscopic stone treatment; the role of these techniques with semirigid ureteroscopy has not yet been characterized.

\section{Author Disclosure Statement}

No competing financial interests exist.

\section{References}

1. Vassar GJ, Chan KF, Teichman JM, et al. Holmium:YAG lithotripsy: Photothermal mechanism. J Endourol 1999;13:181.

2. Chan KF, Vassar GJ, Pfefer TJ, et al. Holmium:YAG laser lithotripsy: A dominant photothermal ablative mechanism with chemical decomposition of urinary calculi. Lasers Surg Med 1999;25:22.

3. Sea J, Jonat LM, Chew BH, et al. Optimal power settings for holmium:YAG lithotripsy. J Urol 2012;187:914.

4. White MD, Moran ME, Calvano CJ, et al. Evaluation of retropulsion caused by Holmium:YAG laser with various power settings and fibers. J Endourol 1998;12:183.

5. Finley DS, Petersen J, Abdelsehid C, et al. Effect of Holmium:YAG laser pulse width on lithotripsy retropulsion in vitro. J Endourol 2005;19:1041.

6. Wollin DA, Ackerman A, Yang C, et al. Variable pulse duration from a new Holmium:YAG laser: The effect on stone comminution, fiber tip degradation, and retropulsion in a dusting model. Urology 2017;103:47-51.

7. Wezel F, Hacker A, Gross AJ, et al. Effect of pulse energy, frequency, and length on Holmium:YAG laser fragmentation efficiency in non-floating artificial urinary calculi. J Endourol 2010;24:1135.

8. Kronenberg $\mathrm{P}$, Traxer $\mathrm{O}$. In vitro fragmentation efficiency of Holmium: Yttrium-aluminum-garnet (YAG) laser lithotripsyA comprehensive study encompassing different frequencies, 
pulse energies, total power levels and laser fibre diameters. BJU Int 2014;114:261.

9. Chew B, Shah O, Sur R, et al. Dusting vs basketing during ureteroscopic lithotripsy-Which is more efficacious? Final results from the EDGE research consortium. J Urol 2016; 195:e407.

10. Alkan E, Turan M, Ozkanli A, et al. Should stone removal be done after stone fragmentation in the management of upper urinary stones? J Urol 2016;195:e407.

11. Kroneberg P, Traxer O. Update on lasers in urology 2014: Current assessment on Ho:YAG laser lithotripter settings and laser fibers. World J Urol 2015;33:463.

12. Eisner BH, Dretler SP. Use of the Stone Cone for prevention of calculus retropulsion during Holmium:YAG laser lithotripsy: Case series and review of the literature. Urol Int 2009;82:356.

13. Gamal W, Mamdouh A. Flexible URS Holmium laser stone dusting vs fragmentation for $2 \mathrm{~cm}$ single renal stone. J Urol 2015;193:e312-313.

14. Schoenthaler M, Buchholz N, Farin E, et al. The postureteroscopic lesion scale (PULS): A multicenter videobased evaluation of inter-rater reliability. World J Urol 2014;32:1033.

15. Macario A. What does one minute of operating room time cost? J Clin Anesth 2010;22:233.

16. Tracey J, Gagin G, Morhardt D, et al. Stone dusting: Outcomes of ureteroscopic lithotripsy using a multi-cavity high-power Holmium laser. J Urol 2016;195:e510.

17. Tracey J, Gagin G, Morhardt D, et al. Flexible ureteroscopy and laser lithotripsy for renal stones using 'popdusting': Comparison of outcomes between traditional dusting settings versus ultra-high frequency settings. J Urol 2016;195:e683.

18. Glickman L, Munver R. Comparison of low power/high frequency Holmium laser settings with conventional settings on ureteral and renal stone fragmentation efficiency. J Urol 2015;193:e888-e889.

19. Scales CD Jr, Saigal CS, Hanley JM, et al. The impact of unplanned postprocedure visits in the management of patients with urinary stones. Surgery 2014;155:769.
20. Haleblian G, Kijvikai K, de la Rosette J, et al. Ureteral stenting and urinary stone management: A systematic review. J Urol 2008;170:424.

21. Rapaport D, Perk AE, Teichman JM. Ureteral access sheath use and stenting in ureteroscopy: Effect on unplanned emergency room visits and cost. J Endourol 2007;21:993.

22. Chew BH, Brotherhood HL, Sur RL, et al. Natural history, complications and re-intervention rates for asymptomatic residual stone fragments after ureteroscopy: A report from the EDGE research consortium. J Urol 2016;195:982.

23. Lee YJ, Bak DJ, Chung JW, et al. Is it necessary to actively remove stone fragments during retrograde intrarenal surgery. Investig Clin Urol 2016;57:274.

24. Schatloff O, Lindner U, Ramon J, et al. Randomized trial of stone fragment active retrieval versus spontaneous passage during Holmium laser lithotripsy for ureteral stones. J Urol 2010;183:1031.

25. Fahmy A, Youssif M, Rhashad H, et al. Extractable fragment versus dusting during ureteroscopic laser lithotripsy in children: Prospective randomized study. J Pediatr Urol 2016; $12: 254$.

Address correspondence to: Brian R. Matlaga, MD, MPH James Buchanan Brady Urological Institute Johns Hopkins Medical Institutions 600 North Wolfe Street, Park 221 Baltimore, MD 21287

E-mail: bmatlaga@jhmi.edu

$$
\begin{aligned}
& \text { Abbreviations Used } \\
\mathrm{CT} & =\text { computed tomography } \\
\mathrm{HF} & =\text { high frequency } \\
\mathrm{KUB} & =\text { kidney }, \text { ureter, and bladder radiograph } \\
\mathrm{LP} & =\text { low power } \\
\mathrm{US} & =\text { ultrasound } \\
\mathrm{YAG} & =\text { yttrium aluminum garnet }
\end{aligned}
$$

